\title{
Castas: Identidades que atraviesan fronteras
}

\section{Nora Lucía Ibarra ${ }^{1}$ Universidad de Buenos Aires}

\section{Ensayo}

Material original autorizado para su primera publicación en Journal de Ciencias Sociales, Revista Académica de la Facultad de Ciencias Sociales de la Universidad de Palermo.

Recepción: 24-03-2020

Aceptación: 25-04-2020

Resumen: El siguiente trabajo pretende visibilizar distintas maneras de concebir a las castas en India y, en particular, a la discriminación en base a ellas. Para ello se procede a problematizar nociones teóricas acerca del sistema de castas, planteando interrogantes sobre los modos en los que las identidades de castas son vividas y concebidas hoy. La existencia de un único sistema de castas vigente desde épocas védicas, la noción de un fenómeno único y exclusivo de la India y la idea de castas como identidades estáticas e inmodificables son algunas de las fronteras que se buscan atravesar. La homologación entre la discriminación racial y la discriminación en base a las castas será uno de los debates en los que más se ahondará. Lejos de ser cuestiones meramente teóricas, se verá cómo la definición que se adopte sobre la naturaleza de la discriminación en base a casta podría ser utilizada como fundamento para forjar políticas públicas desde organismos internacionales hacia India y otros países surasiáticos, como así también en el territorio del Reino Unido, referentes a la diáspora. El accionar de organizaciones internacionales, tales como la International Dalit Solidarity Network y la WCAR, serán actores e instancias claves en tales disputas, presentando diferentes estrategias de combate a esta discriminación.

Palabras clave: identidades; castas; discriminación; estratificación social.

\section{Castes: Identities that cross borders}

Abstract: The following essay aims at shedding light on different ways to conceive caste in India, specifically caste-based discrimination. In order to do so it questions notions on caste as a system and on the way that caste performs in nowadays world. The existence of just a

\footnotetext{
${ }^{1}$ Socióloga de la UBA y maestranda en investigación en ciencias sociales por la UBA. Miembro del Grupo de Trabajo sobre India y Asia del Sur del Comité de Asuntos Asiáticos en el Consejo Argentino para las Relaciones Internacionales (CARI) y miembro de la Cátedra Libre de la India en el Instituto de Relaciones Internacionales (IRI) de la UNLP. Correo electrónico: n.luciaibarra@gmail.com
} 
single caste system dating from vedic times, the notion of a unique phenomenon from India and the perception of caste as a fixed identity are some of the frontiers that this essay will try to cross. The homologation between racial discrimination and caste-based discrimination will be one of the core debates developed in this essay pointing out two opposite view on the matter. Far from being merely theoretical questions, it will be seen how the definition adopted on the nature of caste-based discrimination could be used as a basis to forge public policies from international organizations towards India and other South Asian countries, such as in the territory of the United Kingdom, referring to the Indian diaspora. The actions of international organizations, such as the International Dalit Solidarity Network, and WCAR will be key actors and instances in such disputes, presenting different strategies to combat this type of discrimination not only in India but across its borders.

keywords: identities; caste; discrimination; social stratification.

\section{Introducción}

El siguiente trabajo se propone reflexionar acerca de las castas como identidades que lejos de ser estáticas y estar ancladas a un territorio estatal en particular, atraviesan fronteras. Para ello, sumo la consideración de un elemento lúdico, tal es un dado triangular (que tiene 4 lados, a diferencia de una figura piramidal, que tiene 5), siguiendo la idea de entrelazar el texto con una figura geométrica, desarrollada por Fraisse (2011).

En primer lugar, se abordarán algunas nociones y maneras de entender las castas, aclarando el por qué referirse a ellas como identidades y no como un sistema. Girando el dado hacia un costado, el abordaje del caso del debate llevado a cabo en la World Conference against Racism / WCAR, en Durban durante 2001, ofrece la perspectiva de pensar la identidad de casta como identidad racial en tanto que ambas podrían ser utilizadas como bases para prácticas discriminatorias, evidenciando cómo la frontera entre lo que algunos consideran: "cuestiones internas del Estado nacional Indio", se ve atravesada por la demanda de apoyo de parte de organismos internacionales. En el tercer lado, la consideración de un nuevo debate surgido en torno a la sanción de la Single Equality Bill, en el Reino Unido en 2006, muestra nociones de castas que operan y compiten entre sí, en la diáspora india. (Douglas, 2014). Mientras que un sector considera que las castas son estigmas que se transportan con los migrantes, otros consideran estos marcadores sociales como rasgos culturales que no tienen como concomitante prácticas discriminatorias. Por último, se abordará la labor de la International Dalit Solidarity Network / IDSN, red de organizaciones no 
gubernamentales, que se propone actuar en la esfera internacional como representante de los derechos de los Dalits².

Cabe aclarar que, en este trabajo, las castas son consideradas como categorías socialmente relevantes, que operan en los territorios de distintos Estados-nación, especialmente teniendo en cuenta los conflictos que surgen en cuanto a la discriminación de los Dalits.

\section{Castas: definiciones y formas de entenderlas}

Desde este lado del dado, veremos algunas de las múltiples conceptualizaciones acerca de las castas, sin por ello dar por cerrada la problematización de tal noción. En primer lugar, debe señalarse que el término "casta" no posee un equivalente en sánscrito. Su origen etimológico remite a una categoría utilizada por los portugueses, quienes en el proceso de la expansión ultramarina que protagonizaron, designaron de este modo a los grupos con los que entraron en contacto en India.

Al respecto, Banerjee (2008) sostiene:

La suposición de que la casta ha existido desde su inicio como una institución panindia con una ideología y normas comunes se debe en gran medida a la presencia del influyente concepto de varna (color) en "escrituras" hindúes tempranas. El esquema de varna divide la sociedad en cuatro grupos desiguales y estratificados según su ocupación: brahmanes (maestros y sacerdotes), kshatriyas (príncipes y guerreros), vaishyas (mercaderes y comerciantes) y sudras (trabajadores menores). El varna refuerza la idea de la casta o jati con su origen en el nacimiento, como una jerarquía uniforme, clara e inmutable en toda la India, compuesta sólo por cuatro grupos con la adición ocasional de los dalits, intocables fuera del esquema pero definidos por el mismo. Este esquema explica buena parte de la confusión que rodea a la casta. Para empezar, varna y jati son términos polisémicos cuyos significados se traslapan en gran medida. Además existen diversas teorías en cuanto a la correlación entre los dos (pág. 326).

La autora hace referencia a esta interpretación como una confusión. En parte porque la palabra casta es, como sostiene Khilnani (2004), la descripción que hicieron occidentales perplejos de este sistema y, también, porque una amplia distancia separa los alegatos engañosamente bien definidos del orden de castas y las operaciones reales de lo que es esencialmente un sistema local que actúa por sobre una escala pequeña. Por otro lado, el sistema tampoco concentra riqueza, status y poder exclusivamente en uno de los grupos

\footnotetext{
2 Dalit es una palabra de la lengua marathi que connota el estado de estar abatido u oprimido. Desde los 1970s ha sido usado genéricamente en India para referirse a la población antes considerada como intocable debido a las prácticas de exclusión espacial y social impuestas para con tales personas. Posteriormente la palabra fue y es utilizada en luchas de reconocimiento de derechos, siendo organizaciones de dalits un actor político relevante.
} 
sociales sino que los distribuye entre distintos sectores, generando así las bases para la competencia entre castas. Competencia a la que hace referencia Gupta (2005), buscando resaltar que el esquema fijo, carente de conflicto, jamás tuvo vigencia plena. Se debe destacar que este esquema no operaba de forma igual en el territorio de lo que hoy es la República de la India y que tanto las formas de entender este concepto, como su relevancia en la vida cotidiana, fue fluctuando desde la óptica de la administración colonial y poscolonial. Razón por la cual, resulta pertinente indagar en algunos de estos procesos.

Ganguly (2005) busca aproximarse también a la casta a través de las distintas miradas teóricas que la describieron y sostiene que luego de la independencia de India, en los discursos políticos, la jerarquía tradicional de las castas fue percibida como un significante indisputable del atraso de India. Se podría decir que luego del Motín de los Cipayos en 1857 y con la disolución de la Compañía Británica de las Indias Orientales (al convertirse la India formalmente en una colonia británica), los administradores coloniales pusieron un énfasis especial en respetar las tradiciones y las religiones de la India. Es allí donde cobra mayor fuerza la invención de la tradición india, lo que viene de la mano de una fosilización de procesos dinámicos bajo la mirada orientalista de una sociedad que, al ser catalogada como tradicionalista, se piensa como congelada en el tiempo. La casta emerge como una categoría clave de clasificación, enumeración y control bajo el dominio colonial principalmente mediante dos proyectos de gobernabilidad: la recolección de impuestos sobre la tierra (collection of land revenue) y el censo nacional (All Indian Census) (Ganguly, 2005).

Como indican numerosos trabajos antropológicos, la casta no era en la India precolonial una categoría habitual, ni mucho menos utilizada para la clasificación de las poblaciones. De hecho, el sistema de castas -con excepción de las castas aristocráticas- era muy dinámico y disperso. En distintos territorios existían diversos ordenamientos de castas y distintos nombres para las mismas. Fue necesario, en este caso, todo un proceso de regimentación, fijación, y reificación, bajo la mirada colonial, lo que permitió que una categoría artificial como la casta, emergiera como una identidad social consolidada (Ganguly, 2005).

Los censos que se comenzaron a elaborar bajo el dominio británico, aproximadamente desde 1871, significaron la piedra de toque para la consolidación del sistema de castas, como sistema de clasificación social, y productor de identidades. Los censos, usualmente, se engloban dentro de una tecnología de poder, orientada al gobierno de las poblaciones. Esta tecnología se dirige a la multiplicidad de los hombres, considerando a estos, ya no como cuerpos, sino como masa global. Una tecnología de gobierno que se enfoca en los procesos vitales de las poblaciones (tales como: su natalidad, su morbilidad, su productividad, su relación con el medio geográfico, climático, etc.); en otras palabras "las 
relaciones entre los seres humanos como especie, como seres vivientes, y su ambiente de existencia" (Foucault, 1996, p. 198).

En esta mensuración de las poblaciones indias, iniciada con el All-India Census, se despliega la biopolítica como tecnología de gobierno. La reificación del sistema de castas, y la centralidad que adquiere para el colonialismo británico la configuración de identidades sociales más o menos fijas, hablan de la consideración que adquiere la regulación de los procesos vitales del hombre-especie (Foucault,1966, p. 199), pasando a ser objeto de diferentes iniciativas gubernamentales.

Así, una población clasificada, etiquetada, en la que cada casta adquiere características que le son intrínsecas, sujetas a estimaciones, previsiones, estadísticas y medidas globales; se vuelve objeto del biopoder. No se debe perder de vista lo que estas prácticas deben haber significado, para la sociedad india del siglo XIX, en el marco de un contexto de dominación colonial extranjera.

Pero, así como las identidades se fijan, se generalizan y se vacían de especificidad (Devalle, 2002), también señala que las identidades asignadas pueden ponerse en discusión por parte de los sujetos que fueron vistos como objetos. Es habitual que estos procesos de resignificación y reconstrucción de la identidad de un grupo, conlleven un trabajo tanto interno como externo de pensar y sentir la necesidad de una nominación diferente, de transformar aquella categoría impresa sobre sus cuerpos en otra, de transformar y resignificar esas fronteras "graficadas" sobre los cuerpos de las personas.

En este sentido, la historización del término dalit es un claro ejemplo de ello. La palabra derivó de la lengua marathi, y connota la condición de estar abatido u oprimido. Desde los años 70 del siglo XX, la palabra ha sido usada genéricamente en India para representar las castas que eran consideradas intocables, desafiantes de la hegemonía hindú brahmánica, de las castas altas del norte de la India en el escenario político nacional pos independentista.

Quien primero usó el término en este sentido fue Jyotiba Phule. Sin embargo, fue el auge del Dr. B. R. Ambedkar -en la década del 30 del siglo XX- con su denuncia políticocultural del sistema de castas, (además de su lucha para generar una nueva identidad entre las castas oprimidas), lo que le dio al término Dalit la fuerza y difusión que tiene hasta el día de hoy. En el marco de la búsqueda de una identidad propiamente india para gran parte de los proyectos independentistas, las inequidades basadas en el sistema de castas fueron un problema que generó diversos debates entre intelectuales y políticos como Ghandi, Jawarhal Nehru, su padre Motilal y el mismo Ambedkar (entre otros). 
En la concepción del nacionalismo como un proyecto desarrollista, la ideología y la práctica de las castas ha sido considerada como anómala y perjudicial, tanto para la nación, como para los ciudadanos, así como también para la construcción de una ciudadanía nacional. En la redacción de la Constitución nacional se planteó la necesidad de poner en práctica políticas sociales de discriminación positiva, mediante las cuales se pretendió otorgar, a los miembros de grupos considerados desfavorecidos, una ayuda que permitiese paliar la desigualdad de oportunidades. Estas políticas ya se habían puesto en práctica durante el colonialismo británico mediante un acta llamada: The Government of India (Scheduled Castes) Order, en 1936, por medio de la cual se clasificaba aquellos grupos que serían considerados como parte de las llamadas "Depressed classes". Luego, en los artículos 341 y 342 de la Constitución, estos grupos fueron definidos como SC (Schedule Caste), ST (Schedule Tribes). Más tarde, en la Comisión de Mandala en 1979, como OBC (Other Backward Classes) (Ganguly, 2005).

Esta tensión, generada entre los intentos nacionalistas-estatistas por hacer que las diferencias de castas sean distintas (a través de la retórica de los derechos y la ciudadanía), junto a las conflictividades derivadas de la marcación de los cuerpos de las castas subalternas -como SC O OBC- fue advertida por Partha Chatterjee refiriéndose a ella como: ambigüedades de legitimación del proyecto estatista nacionalista (en Ganguly, 2005). Al pretender eliminar a la casta, como una categoría válida para determinar las trayectorias personales de los/as sujetos, se terminó sobreimprimiendo la pertenencia a alguna de ellas a todos sujetos que fueron discriminados en base a ella. Es decir que, con el afán de combatir la desigualdad generada por el sistema de castas, se resalta la importancia de la pertenencia a castas generando que aquello que se buscaba eliminar como sistema de referencias, se convierta en una referencia o marcador aún más importante que antes.

Ahora bien, cada estado provincial tuvo que afrontar la tarea de establecer los parámetros mediante los cuales se determinaría quienes formarían parte de estos grupos. Como la intocabilidad tuvo diversas manifestaciones en diferentes partes de la India, estaba muy lejos de ser obvio qué castas debían ser incluidas en la Lista de la Constitución (Galanter, 1984 citado en Gupta 2005). En cualquier caso, más o menos el 17\% de los escaños de las instituciones educativas y de los trabajos del sector público estaban reservados para las SC y, del mismo modo, alrededor del 7\% para las Scheduled Tribes (ST).

El efecto que tuvieron estas políticas no repercutió sólo sobre la sobre identificación antes mencionada, sino también sobre la dinamización del sistema o bien, según Dipankar Gupta (2005), significó la ruptura del mismo, en pedazos. El autor afirma que la jerarquía ritual, pura, se mantiene sólo cuando es respaldada por la riqueza y el poder (Gupta, 2005). Actualmente, las características morfológicas de la estructura contemporánea agraria inhiben 
tanto las desigualdades acumulativas como la dominación concluyente de algún sector de la sociedad. La riqueza ya no respalda la dominación de un sector en particular. Y la emergencia de pequeños y medianos propietarios de tierras, que constituyen el $34 \%$ de la población nacional, pero controlan el $51 \%$ de la tierra, es uno de los factores que -para Gupta- explica la ruptura mencionada y la emergencia de las identidades de casta.

Las castas son, principalmente, para Gupta (2005), entidades discretas, con una profunda tradición ideológica. La noción de identidades de casta se vincula con afiliaciones identitarias que se pueden visualizar actuando de manera más transparente que en el sistema de castas mediante competencias por espacios de poder. Esto no implica que se pueda encontrar, en los procesos electorales, por ejemplo, una ecuación directa entre la pertenencia a una casta y el voto hacía lideres o partidos que alegan representar la misma. La representación política misma se encuentra en disputa, entrando en juego otro tipo de afiliaciones y de racionalidades propias de una democracia capitalista con un amplio desarrollo de los medios de comunicación y una vasta diversidad lingüística y religiosa.

\section{La casta como raza}

Otro giro representa la consideración del debate sobre las categorías referidas especialmente a identidades, a adscripciones o imposiciones identitarias. En lo que ocupa a este trabajo, se observa cómo la casta atraviesa las fronteras del Estado nacional indio para formar parte de una conferencia -de escala internacional- llevada a cabo en Durban durante el 2001 (ciudad costera de la provincia sudafricana KwaZulu-Natal). Se hace referencia a la Conferencia Mundial en contra del Racismo (WCAR, por sus siglas en inglés). Allí, distintos grupos de activistas plantearon su parecer sobre la casta como un marcador socio-político utilizado para fundamentar prácticas discriminatorias en India, tanto por el Estado como por otras instituciones de la comunidad civil.

Las WCAR, son eventos internacionales establecidos por la Organización de las Naciones Unidas. El primero de estos encuentros se llevó a cabo en 1978 en Ginebra (estuvo enfocado principalmente en el apartheid sudafricano). La Conferencia de Durban se llevó a cabo entre el 31 de agosto y el 7 de septiembre del 2001.

Ante la propuesta de incluir el problema de la casta en la WCAR, el gobierno indio declaró estar en contra de internacionalizar el tema por diversos motivos. Por un lado, se consideró que la casta y la raza son dos entidades disímiles e incongruentes; por el otro, la postura oficial sostuvo que fue consciente de las necesidades de las SCs y que tomó las medidas legislativas para la emancipación de estos grupos a través de la abolición de la intocabilidad, además de activar distintas políticas de discriminación positiva junto a planes 
de asistencia económica (Pinto, 2001). Y, por último, también se argumentó que la internacionalización del problema no traería otra consecuencia más que estigmatizar a la India como un país culturalmente retrógrado, contribuyendo al desconocimiento de las capacidades nacionales para resolver el problema de la discriminación con base a la casta.

Es en este último punto en el que Dipankar Gupta hace hincapié en una nota escrita para el diario The Times of India. Con cierta ironía destaca que, incluso desde la homologación entre casta y raza, esta no es aceptada por los activistas dalits y tampoco por los académicos que los apoyan. Por esta razón, es que se decide presentar el tema para que sea abordado en la Conferencia de la ONU. Resulta relevante tener presente a Gupta (2004), cuando señala que las castas hoy actúan en un contexto diferente en el cual pueden expresar sus políticas identitarias, pues las transparencias contemporáneas han arrojado luz sobre aspectos de la casta que antes permanecían oscuros. Para el autor, la casta no ha cambiado, pero las potencialidades que yacían en este sistema de estratificación social ahora se encuentran abiertas y a la vista.

En una nota publicada en el diario The Hindu, el 25 de septiembre del 2001, por el ex gerente general de la India Railways, V. Ramanathan, el autor destaca que la homologación entre casta y raza surgió en el siglo XIX cuando lingüistas notaron que la descripción del sistema en sanscrito era varna dharm. Al ser varna comúnmente traducida como color, dichos lingüistas interpretaron que el sistema de castas tenía como principio de jerarquización de los grupos el color de la piel. Pero varna también significa descripción, y el color al que se hace referencia con la palabra varna está íntimamente relacionado a la ocupación de los segmentos del sistema, de manera tal que los ksatriyas es rojo por el contacto que los guerreros tienen con la sangre; los vaisyas tendrían el color amarillo por el color del dinero con el que comercian, los brahmanes -por ser su deber llevar hacia la pureza- son representados por el color blanco, y los sudras -que se encuentran impedidos de acceder a la luz del conocimiento- por el negro. De nuevo vemos presente la confusión que mencionaba Banerjee (2008) como una concepción hegemónica y de gran difusión.

Ahora bien, quienes planteaban que se realizase dicha homologación han presentado razones por las cuales la casta no es igual a la raza. Se sostiene que las prácticas y los discursos generados por la discriminación en base a ambas categorías de visión, clasificación y división, son extremadamente similares y es por ello que el tema debería ser tratado en la WCAR. En otras palabras, los activistas y académicos dalit quisieron equiparar a la casta, no solo desde el plano analítico- conceptual de la razón, sino también legalmente (Visvanathan, 2001). 
A continuación, se revisarán algunas de las razones centrales por las cuales, según los activistas dalits, el problema debió ser discutido en la CWAR. Posteriormente, se verán los argumentos presentados por los intelectuales y activistas dalits en la Conferencia de Delhi.

Como primera razón, se mencionó, según Visvanathan (2001), el hecho de que la Conferencia trabajara sobre el tema de la discriminación basada en la ocupación heredada. La casta entra en este esquema debido a la imbricación de los sistemas varna y jati como se vio anteriormente. Este punto, por otro lado, sería refutado por parte de las autoridades gubernamentales de India debido a que se desconocía la vigencia y los efectos de las políticas de discriminación positiva, pues los cupos no otorgaban sólo oportunidades para quienes desearan elevar su nivel educativo formal, sino que también existían cupos en los puestos de trabajo estatales.

Luego, y este es un punto largamente desoído, los grupos dalits se rehusaban a aceptar que la casta sea un sistema exclusivo de la India, ya que operaba también en otros países del sur de Asia como Nepal, Bangladesh, en Japón (con los Eta o burakumin), en Corea, (con los Osu), en Nigeria y en otras comunidades que has sido afectadas por la "intocabilidad". Así, debido a su carácter internacional, fue relevante que el problema haya recibido un abordaje dentro del marco de un organismo de esa índole. En cuanto al último argumento, cabe la pregunta sobre qué es lo que se tomó en consideración realmente: la casta en sí misma o a la condición específica de "intocabilidad".

Vale la pena destacar que existe un acuerdo entre el Comité de Organizaciones No Gubernamentales y la Coordinación Regional Asia-Pacifico de Organizaciones No Gubernamentales de la WCAR, por el cual se asegura que la discriminación en base a casta - que afecta a los dalits - es una clara violación a los derechos humanos y que debe ser tratada en la WCAR (Pinto, 2001). Siendo India miembro de la ONU, ha firmado y ratificado un sinnúmero de acuerdos internacionales claves en materia de derechos humanos, que incluyen la Convención de Eliminación de Toda Forma de Discriminación Racial (CERD, por sus siglas en inglés). Por el hecho de firmar esta convención India se encuentra comprometida, no sólo a erradicar toda forma de discriminación racial, sino también la discriminación en base a la ocupación heredada. Este tal vez sea el punto más fuerte del argumento en lo que se refiere a materia legislativa, porque se presenta como un claro antecedente de una voluntad política estatal para combatir este tipo de discriminación y, a su vez, se convierte en una prueba del abordaje conjunto que se le debe dar a la discriminación racial al igual que otros tipos de discriminaciones ampliamente definidas bajo el régimen de ocupación hereditaria. Cabe destacar que, en 1996, en el Informe estatal sobre India de la CERD, el organismo afirmó que, si bien la casta no es equivalente a la raza, la discriminación 
en base a casta cae en el ámbito de la injerencia de la Convención, debido a que en el artículo primero se incluye la discriminación en base a la ascendencia (Pinto, 2001).

Por otra parte, los activistas dalits consideraron que el hecho de llevar el reclamo a la WCAR generaría una mayor consciencia internacional en torno a la problemática de la casta en India. Este punto es el eje central de la crítica que desplegó Gupta (2001) a la iniciativa y que será una de las cuestiones a abordar en este trabajo cuando se consideren las consecuencias de la reunión del WCAR en Durban.

Un último argumento que vale la pena mencionar es que la violación de los derechos humanos no está permitida para ningún país y, además, siendo que el Estado nacional indio no dió respuestas satisfactorias para el grupo de los dalits, ellos -como damnificadospresentaron su caso frente a las autoridades internacionales. En este punto se puede ver, por un lado, aquello que criticaba Gupta acerca de la internacionalización de todos los conflictos y, por otro lado, como la casta pasa a ser percibida como un sistema que opera de la misma forma que la raza.

El artículo 341 de la Constitución nacional india ocupa un lugar central en cuanto a la equiparación de castas y razas. De hecho, en este precepto constitucional se basan quienes arguyen que la casta es peor que la raza. En el mismo, se demostraría que, dentro de las Scheduled Caste, se incluyen también las razas, con lo cual, la discriminación en base a la casta no sólo sería una forma de discriminación racial si no todavía más que ello.

El texto constitucional reza:

341. Scheduled Castes - (1) The President may with respect to any State or Union territory, and where it is a State after consultation with the Governor thereof, by public notification, specify the castes, races or tribes or parts of or groups within castes, races or tribes which shall for the purposes of this Constitution be deemed to be Scheduled Castes in relation to that State or Union territory, as the case may be.

(2) Parliament may by law include in or exclude from the list of Scheduled Castes specified in a notification issued under clause (1) any caste, race or tribe or part of said a notification issued under the said clause shall be varied by any subsequent notification (citado en Oommen et al, 2001).

Por otra parte, dos medidas legislativas dan cuenta de que la discriminación en base a casta es más peligrosa y violenta que la discriminación racial y, a su vez, demostrarían la inefectividad de las medidas tomadas por el Estado. Estas son: la Protection of Civil Rigths Act de 1976 (previamente llamada The Removal of Untochability Act, 1955) y la SCs/STs (Prevention of Atrocities) de 1989 (Oommen et al, 2001).

Finalmente, en la CWAR la discriminación racial fue ampliamente definida como la discriminación basada en motivos de raza, color, ascendencia y origen nacional o étnico. Se 
hizo especial referencia a la India, país que se negó a aceptar la ascendencia como motivo de discriminación intrínseca al sistema de castas, sosteniendo que el término sólo se aplicaba en relación con la discriminación racial; siendo que la Convención - 1996- observó que la situación de las SCs y STs entraba en el marco en su ámbito (Macwan, 2001).

Como destaca Macwan (2001) -coordinador de la Campaña Nacional de los Derechos Humanos de los Dalits (National Campaign on Dalit Human Rights)- la internacionalización de la discriminación en base a la casta ha ayudado a la causa gracias a los medios internacionales y nacionales e, incluso, gracias a la negativa del gobierno de la India en tratar la temática en Durban. Desde la perspectiva del autor, nuevos desafíos se abren a partir de esta victoria del primer round, como lo llama Naunidhi Kaur (2001), en Frontline.

Esta victoria, no obstante, debe ser matizada porque, en el caso particular de la CWAR, el IV evento -que tenía como objetivo revisar los acuerdos logrados y firmados en Durban (y que por ello fue conocida como Durban II)- fue boicoteado en Ginebra por varios países, cada uno de ellos con distintos motivos que no son el objeto de este trabajo. Asimismo, se debe destacar que desde la CERD se continuó monitoreando el abordaje efectivo que realizara el Estado indio en materia de discriminación por casta (Ibarra y Navarro, 2013).

Si se consideran los resultados alcanzados por la convención realizada en Ginebra, en febrero de 2007 a la luz de los activistas dalits, queda en evidencia que ellos no tuvieron éxito, pues los delegados del gobierno de India insistieron en que el Estado estaba comprometido en erradicar toda forma de discriminación en base a la casta y en que, precisamente por ello, no generarían informes específicos para la Conferencia. Se verá en el siguiente apartado, cómo la decisión tomada por la CWAR en Durban influyó en la decisión que consensuara el Parlamento británico en 2010. (Ibarra y Navarro, 2013)

\section{La casta en la diáspora británica}

El siguiente lado del dado, o ejemplo en este caso, tiene que ver con el debate que se llevó a cabo en el marco de la discusión parlamentaria sobre el Single Equality Bill, un recurso legislativo para combatir la discriminación en el Reino Unido. Este recurso formó parte de una propuesta electoral del partido Laborista en el 2005 y comenzó a trabajarse en el 2007 para ser sancionado, finalmente, en octubre de 2010. Es durante este período que surge la propuesta de parte de miembros del parlamento (como Jeremy Corbyn y Rob Marris), sobre prohibir la discriminación en base a castas (Chahal, 2008).

Frente a esta propuesta el Hindu Council UK publicó un informe sobre el sistema de castas, escrito por Dr Raj Pandit Sharma y contestado por Chahal (2008) -en tanto presidente 
de la Federation of Ambedkarian and Buddhist Organizations (FABO)- con esto, se pretendió visibilizar las voces de organizaciones que actúan en el Reino Unido y que tienen una postura diferente a la del Hindu Council en lo que respecta a la vigencia y el origen del sistema de castas tanto en India como en la diáspora india.

Chahal (2008) define al sistema de castas como un concepto religioso en el cual cada casta rechaza cualquier conexión con otras castas y no comparte ni la comensalidad, ni habilita los casamientos entre ellas. El sistema estaría fijado de tal manera que la pertenencia a una casta sería hereditaria y se reconocería de un vistazo por la apariencia, por la forma de vestir, por el nombre, por la forma en la que se saluda a otro, por los patrones generales de comportamiento y por el uso del idioma. Claramente puede verse aquí presente, de nuevo, el esquema mencionado por Banerjee (2008). Al considerar que esta es la descripción correcta del sistema de castas, se desconoce las transformaciones del sistema, su diversidad a lo largo y ancho del territorio indio y, a su vez, se pasa por alto la imbricación entre dicho sistema y otros modos de estratificación social como -por ejemplo- la sociedad de clases.

Es bastante llamativo que en su respuesta al Hindu Council, Chahal (2008), tenga otra consideración de lo que fue uno de los puntos claves en el debate de Durban, esto es el hecho de que el sistema de castas se pone en práctica mediante la imposición de la ocupación hereditaria. Al considerar que el sistema de castas es una institución religiosa, rechaza la explicación según la cual estos grupos fueron constituidos en base a las ocupaciones de los mismos. La interpretación de la historia del subcontinente arriba a la conclusión de que las masas budistas -conquistadas por los hindúes- son convertidas en Intocables. Más allá de la precisión o no de dicho relato, tema que no hace a los ejes de este trabajo, es interesante destacar la clara influencia de la teoría de las etapas históricas realizada por Ambedkar, según la cual, existirían tres etapas previas a la colonización británica. La primera sería la India del Brahmanismo, o la sociedad Aria del período védico; (en verdad, de acuerdo con Ambedkar una fase barbárica). La segunda, la India del budismo, con los imperios Magadha-Maurianos personificando la cima de la civilización humana, donde todos los indios eran tratados como iguales y con dignidad. Y la tercera, la India de la contra revolución hindú, proclamado históricamente con la llegada al poder de Pushyamitra Sunga y asociada con Manu, el legislador hindú que legitimó y codificó el sistema de castas (en Ganguly, 2005).

Este relato acerca del origen de las castas es también utilizado como argumento, de parte de Chahal (2008), para refutar la idea del Hindu Council, según la cual las castas fueron impuestas por gobernantes extranjeros. $Y$ es también este relato acerca del origen de las castas una interpretación que desconoce los procesos que afectan al sistema de castas en el subcontinente indio a partir de su colonización por parte de la corona británica. 
Sin embargo, si se realiza una historización de las maneras de interpretar y lidiar con la problemática de las castas, tomando en cuenta a las figuras centrales de la lucha independentista india, Gandhi consideraba que el objetivo del sistema de castas (o Chatturvarna) era evitar la lucha de clases (Chahal, 2008). Al fijar los deberes y la ocupación de una persona, desde antes de nacer, ella debía responder a la profesión o el oficio que le hubiera sido asignado. Adoptar un sistema social europeo occidental significaba que los hindúes debieran renunciar al principio de la ocupación hereditaria, que era el alma del sistema de casta; lo cual podría haber generado un gran caos social. Un debate histórico que muestra las distintas concepciones que tuvieron Gandhi y Ambedkar es aquel que da cuenta sobre las negociaciones para que los dalits se constituyeran como un electorado separado tras la firma del Poona Pact en 1932. (Chahal, 2008)

En cuanto a las políticas de discriminación, destinadas a las SCs, los planes económicos y la legislación tendiente a condenar las prácticas discriminatorias hacia estos sectores, Chahal (2008) se manifiesta completamente en contra de la renovación de las políticas de discriminación positiva. Considera que aquellas medidas, tomadas inicialmente para actuar solo por el lapso de diez años, han generado efectos adversos durante su prolongación, volviéndose funcionales a los intereses de partidos políticos, sobre todo en lo que respecta a las alianzas de castas. De manera concluyente, el autor sostiene que la implementación de las políticas de cupos reservados -en las instrucciones del ejecutivo- que se somete con frecuencia a la intervención judicial, no ha logrado alcanzar el objetivo deseado.

El desarrollo de condiciones para que las castas puedan contar con igualdad de oportunidades es un objetivo que no ha sido aun completamente logrado. Pero la aplicación de estas medidas ha llevado a que se generen situaciones que no pueden ser comprendidas negando, o disminuyendo, su impacto. Los fenómenos que Chatterjee (2008) denominaba ambigüedades de legitimación, han extendido situaciones y usanzas que, comúnmente, se conocen como creamy layer, donde abundan fondos disponibles para la población dalit que terminan siendo usados para la cooptación de votos o para recrudecer prácticas discriminatorias entre espacios compartidos por personas de diferentes castas.

La intocabilidad es otra práctica de segregación espacial y social que se materializa en el rechazo a la entrada de dalits a ciertos lugares, como ser: templos, instituciones educativas o sitios donde habitan personas de castas "superiores". También incluye la prohibición de la comensalidad entre personas de otras castas (entre ellas los dalits); en la prohibición del uso de utensillos o recipientes que puedan contener agua o comida, grifos de agua e, incluso, en el contacto directo con personas dalits. 
Es cierto que la intocabilidad no puede ser ejercida en todo su rigor en ciudades sobrepobladas por las limitaciones que impone el espacio físico (Ramanathan, 2001), pero esto no implica que no se continúen ejerciendo algunos de estas prohibiciones con cierto tipo de adaptaciones. Varias organizaciones que luchan por los derechos de los dalits, han denunciado que en las aulas de los colegios, los estudiantes dalits son forzados a sentarse en un rincón, separados del resto de los estudiantes u obligados a realizar la limpieza de los baños de la institución.

En líneas generales, en los debates previos a la independencia, existía un acuerdo acerca de la prohibición de la práctica de la intocabilidad, luego esto fue incorporado en el artículo 17 de la Constitución nacional. Se instituyó así que esta práctica de segregación espacial, discriminatoria, puede ser penada por ley. El grupo de académicos y militantes reunidos en Delhi para la redacción del documento "Caste is Race plus", con vistas a ser presentado como parte del debate en Durban, abordó la persistencia de este tipo de delito e indagó por las diferentes formas de discriminación. Con esto, pretendió dar cuenta de que la sanción de la ley no era suficiente para aseverar que la práctica de segregación haya quedado efectivamente erradicada.

Chahal (2008) afirma que la negación del Hindu Council de la vigente incorporación de la discriminación de castas se debe a la iniciativa del profesor Dipankar Gupta de Jawharlal Universidad Nehru de la India, quien compareció ante el órgano de la ONU, el CERD (Comité a favor de la Eliminación de la Discriminación Racial), el 23 de febrero de 2007, afirmando que no hay paralelismo entre la discriminación racial y la discriminación en base a castas. Su testimonio fue duramente criticado y rechazado por los miembros del CERD.

¿Es la intocabilidad un rasgo inherente del sistema de castas?, Chahal (2008) considera que sí y es por ello por lo cual, no sólo apoya la inclusión de las castas como una de las bases para las prácticas discriminatorias en el Single Equality Bill, si no que -ademásconsidera que al erradicar la intocabilidad se estaría erradicando el sistema de castas. Frecuentemente, a lo largo del texto, se hace una analogía entre este sistema y el Apartheid. De hecho, existe una larga cita de quien en ese entonces era el primer ministro de India, Manoham Singh, desde la cual él también despliega una analogía para comprometerse en la erradicación de la intocabilidad que persistía en su país. En este sentido, el premier propuso declarar los años 2007 y 2008 como años libres de Intocabilidad y Atrocidades. Tanto el apartheid como la vigencia de la discriminación en base a castas son pensadas como violaciones de los derechos humanos, trabas para el desarrollo de la democracia a nivel internacional. A lo largo de todo el texto se puede ver que el autor sostiene que aquellos hechos no son únicamente una cuestión interna del Estado en donde suceden, si no que involucran también a la comunidad internacional para tomar cartas en el asunto. La 
participación de la comunidad internacional puede tramitarse de diversas formas, desde el apoyo a organizaciones locales, pasando por la presentación de reclamos en organismo internacionales o participando en distintos organismos (como ONGs, partidos políticos e incluso mediante redes de diplomáticas).

Una idea central en la discusión que se despliega es también aquella que versa sobre la práctica de la intocabilidad en el Reino Unido. Mientras que el Hindu Council, niega que hayan recibido denuncias en ese sentido, Chahal (2008) presenta una serie de casos ocurridos allí; incluso procede a explicar la existencia de distintas iglesias o templos para las diferentes castas, en base a la expulsión de dalits de estos edificios. Se cita aquí una frase de Ambedkar mediante la cual explica que allí adonde vaya un/a indio/a, viaja con él o ella el sistema de castas. Esta idea nos lleva a pensar que las castas se configuran como categorías de percepción que moldean el comportamiento propio y el esperado por personas de acuerdo a su casta. El hecho de que estas categorías deban ajustarse a nuevos contextos, de lo rural a lo urbano, por ejemplo, no implica que dejen de tener un ejercicio real en la cotidianeidad de las personas. Esto también otorga un sentido adicional a la importancia de la participación de agrupaciones dalits en Durban.

\section{La IDSN: un actor internacional}

Por último, se considerará la red de organizaciones de la sociedad civil, llamada International Dalit Solidarity Network. La misma tiene como propósito llevar a cabo trabajos a escala global para eliminar la discriminación en base a casta y formas similares de discriminación basadas en la ocupación y en la descendencia. Esta red está compuesta por otras redes de solidaridad dalit en Europa, socios internacionales y nacionales, investigadores y plataformas de apoyo y defensa en los países afectados por la problemática. La sede central, debido a que allí están las oficinas administrativas, se encuentra en Copenhaguen, Dinamarca y fue fundada en el 2000.

La red mantiene lazos con la ONU, la Unión Europea y otras instituciones multilaterales. Como objetivos específicos de su accionar esta red se propone:

- Apoyar los derechos de los dalits ejerciendo influencia en las políticas y prácticas de gobiernos y organizaciones e instituciones internacionales.

- Monitorear las medidas que apuntan a combatir la discriminación en base a casta.

- Trabajar hacia el reconocimiento de los Derechos Humanos de los dalit y contribuir a la lucha contra la discriminación global mediante la concientización y el apoyo a iniciativas solidarias. 
- Facilitar las intervenciones internacionales sobre derechos de los dalit, frente a la Comisión Europea y el Parlamento Europeo, los mecanismos de derechos humanos de Naciones Unidas, la Organización Internacional del Trabajo (OIT) y otros.

- Crear y mantener una base de recursos que facilite y fortalezca las funciones de solidaridad y representación, especialmente en Naciones Unidas, la OIT y otros organismos multilaterales e instituciones financieras tales como el Banco Mundial y el Banco Asiático de Desarrollo.

Dentro de la estructura de la red, se pueden distinguir: plataformas en el sur de Asia, en países afectados por la discriminación en base a castas; organizaciones que forman parte de la red y se sitúan en Europa; asociados internacionales; asociados nacionales e investigadores asociados.

En cuanto a los países de Asia en los que se encontraría presente el fenómeno de la discriminación en base a casta, los mismos serían India, Nepal, Pakistán y Bangladesh. Esta visión transnacional de la problemática, como anteriormente se comentó, también fue presentada como un argumento para que la CWAR trabajase el tema, ya no como una cuestión específica y exclusiva de la India, si no como algo social y culturalmente relevante para distintos países del Sur de Asia.

Algunas de las actividades realizadas por la IDSN que resultan más interesantes son las que hacen foco en las empresas que establecen relaciones con los países del Sur de Asia antes mencionados. Para evitar la complicidad de la empresa en cuanto a la discriminación, la red no solo provee a las compañías una herramienta web para que ellas puedan identificar y prevenir la discriminación de dalits, sino que también estableció un marco normativo ISO 26000 por el que se certifica las firmas cumplan con los estándares de responsabilidad social, combatiendo específicamente la discriminación entre sus empleados.

\section{Conclusiones}

La idea de pensar en las castas como identidades que atraviesan fronteras, pretendió darle textura a un fenómeno que fue -y es- frecuentemente simplificado. Si bien queda mucho camino aún por recorrer para llegar a conocer en profundidad la vigencia de las castas, puede ser constructivo tomar el dado propuesto al comienzo de este escrito y echarlo a girar.

Por un lado, quedó en evidencia que las castas pueden ser vistas como un fenómeno identitario que dista mucho de ser aquel marcador social resultante de una mera jerarquía estática y atemporal. Las castas, más allá de dar cuenta sobre la persistencia de tradiciones antiguas, también se revelan como un terreno fértil desde donde indagar sobre la 
construcción identititaria de la llamada India Antigua, en los efectos del colonialismo y en las particularidades de la democracia india.

Visto desde otro ángulo, por los efectos que tiene en distintas esferas de la vida de las personas, la discriminación en base a casta se puede pensar como una discriminación racial en el marco de discusiones acerca de cómo combatir, eliminar y concebir distintos tipos de prácticas discriminatorias en la esfera internacional. $Y$ aquí, también, vale la pena desmitificar aquella asociación de las castas como fenómenos exclusivamente vigentes en la India; desafiando aún más lo referente a la vigencia de la discriminación en base a casta, es posible pensar cómo vive la diáspora india sus identidades de casta. Para ello, este trabajo abordó al caso de la Single Equality Bill, para rescatar los debates que se produjeron, paralelamente, dentro de distintas organizaciones de la diáspora en el Reino Unido.

Por último, la mención del caso de la International Dalit Solidarity Network, permitió revisar las iniciativas que, de forma mancomunada, intentan afrontar la problemática de la discriminación por casta desde una lógica de capilaridad que incluye a entidades públicoprivada, tanto de Europa como del Sur de Asia.

Este trabajo, entonces, se propuso esclarecer algunas de las fronteras que atraviesan las identidades por casta, invitando a reflexionar los horizontes de posibilidad existentes para erradicar las prácticas de discriminación asociadas a estos entramados culturales.

\section{Referencias bibliográficas}

Banerjee, I. (2008). ¿Existe un sistema de castas? Estudios de Asia y África, XLIII, 2, 325382.

Chahal, C. (2008). The Evil of Caste: The Caste system as the largest systemic violation of Human's Rights in Todays World [Reporte de la FABO, The Federation of Ambedkarite and Buddhist Organizations UK[. Recuperado de http://ambedkar.nspire.in/The\%20Evil\%20of\%20Caste\%20by\%20Chanan\%20Ch ahal.html

Chatterjee $P$ (2008). La nación en tiempo heterogéneo, y otros estudios subalternos. Buenos Aires: Siglo XXI Editores.

Devalle, S (2002). Compiladora y Autora. Identidad y Etnicidad: continuidad y cambio. México DF: El Colegio de México- Centro de estudios de Asia y África. 
Foucault, M (1996). Genealogía del Racismo. Buenos Aires: Editorial Altamira.

Fraisse, G. (2011). Del Consentimiento. Santiago: Editorial Palinodia.

Ganguly, D. (2005). Caste, colonialism and counter-modernity: Notes on a poscolonial hermeneutics of caste. New York: Routhledge.

Gupta, D. (Ed.) (2004). Caste in question: identity or hierarchy?. New Delhi: Sage.

Gupta, D. (2005). Caste and politics: identity over system. Annual Review of Anthropology, 21, 409-427.

Ibarra, N.L. y Navarro, L. (2013). Ser Dalit: identidades políticas, sistema de castas y racismo, en la India actual. VII Jornadas de Jóvenes Investigadores. Instituto de Investigaciones Gino Germani, Facultad de Ciencias Sociales, Universidad de Buenos Aires, Buenos Aires. Recuperado en: https://www.aacademica.org/000076/17.pdf

Khilnani, S. (2004). The idea of India. London: Penguin Books.

Pinto, A. (2001). UN Conference against Racism: Is caste race? Economic and Political W eekly, 36(30), 2817-2820.

Visvanathan, S. (2001). The Race for Caste: Prolegomena to the Durban Conference. Economic and Political Weekly, 36 (27), 2512-2516.

\section{Documentos consultados}

\section{Diarios y revistas}

Beteille, A (2001, 10 de marzo). Race and caste. The Hindu. Recuperado de http://hindu.com/2001/03/10/stories/05102523.htm

Gupta, D. (2001, 19 de agosto). Caste is not Race: But, Let's Go to the UN Forum Anyway. The Times of India. Recuperado de http://timesofindia.indiatimes.com/editpage/Caste-is-not-RacebrBut-Lets-Go-to-the-UN-ForumAnyway/articleshow/1229264687.cms

Kaur, N. (2001,6 de julio). Caste and race. Frontline. India's National Magazine. ArcivesBook Info18 (13). Recuperado de http://www.frontline.in/static/html/fl1813/18130950.htm

Ramanathan, V. (2001, 25 de septiembre). Caste, race and Durban Conference. The Hindu. Recuperado de http://www.hindu.com/2001/09/25/stories/13250541.htm

\section{Otros}


Constitution of India. Recuperado de https://www.india.gov.in/sites/upload_files/npi/files/coi_part_full.pdf

Douglas, P (2014). The Equality Act 2010: caste discrimination. Recuperado de https://commonslibrary.parliament.uk/research-briefings/sn06862/

International Dalit Solidarity Network. (2007, 23 y 26 de febrero). Statements and Reflections on CERD'S Review of India's periodic report, Ginebra. Recuperado de http://idsn.org/fileadmin/user_folder/pdf/Old_files/un/pdf/Responses_CERD_India. pdf

Macwan, M. (2001). (Un)Touchable in Durban. Recuperado de http://www.indiaseminar.com/2001/508/508\%20martin\%20macwan.htm

Oommen, T. K et al (2001). Durban Conference must discuss Caste issue. Recuperado de http://www.ambedkar.org/News/Durbanconference.htm

Protection of Civil Rights (PRC) Act, 1955. Recuperado de https://indiacode.nic.in/handle/123456789/1544?view_type=search\&sam_handle $=123456789 / 1362$

Report of the World Conference against Racism, Racial Discrimination, Xenophobia and Related Intolerance (2001). Recuperado de https://www.oas.org/dil/afrodescendants_Durban_Declaration.pdf

The Government of India (Scheduled Castes) Order. 1936

The Scheduled Castes and the Scheduled Tribes (Prevention of Atrocities) Act, 1989. Recuperado de https://indiacode.nic.in/handle/123456789/1920?view_type=search\&sam_handle $=123456789 / 1362$ 